\title{
Session VI
}

\section{Abundance Surveys and Projects in the Era of Future Large Telescopes}




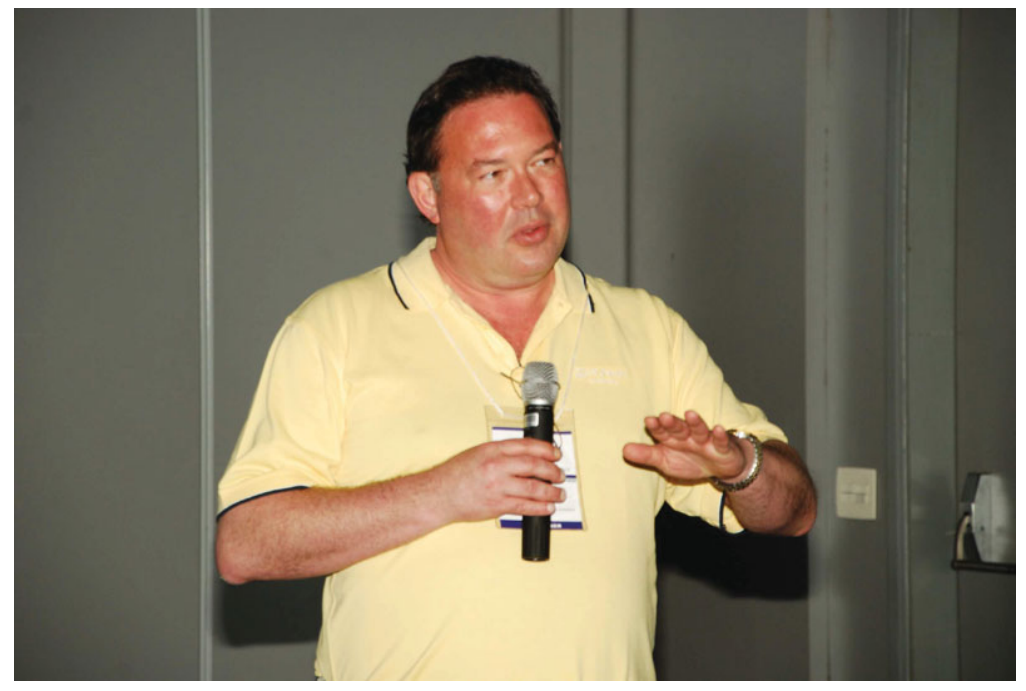

Tim Beers during his talk.

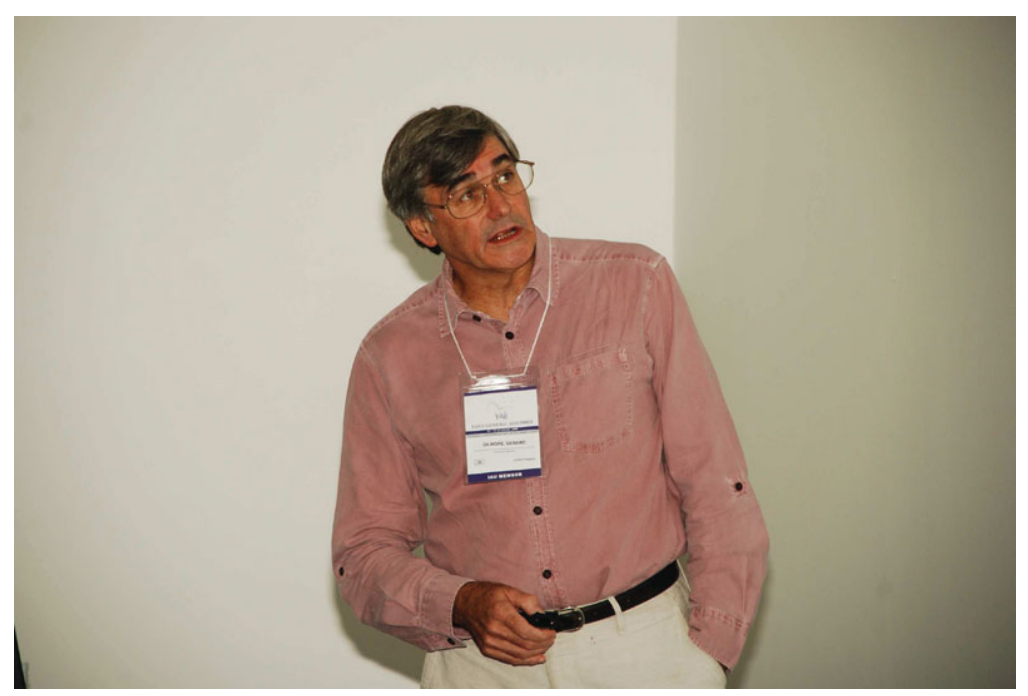

Gerry Gilmore during his talk. 


\title{
Instrumentation in the ELT era
}

\author{
Luca Pasquini ${ }^{1}$ \\ ${ }^{1}$ ESO Karl Schwarzschild Str. 2 D-85748, Garching bei München , Germany \\ email: Ipasquin@eso.org
}

\begin{abstract}
I review the instrumentation plans of the future ELT projects, with particular emphasys on one category of instruments which will be particularly relevant for this Symposium community: high resolution spectrographs.
\end{abstract}

Keywords. Instrumentation, high-resolution spectroscopy

\section{Introduction}

After the great excitement produced by the wealth of high quality data from the $8 \mathrm{~m}$ class telescopes, an even more exciting perspective is opening for ground based astronomy: the era of the Extremely Large Telescopes (ELTs). There are currently three projects which a funded study phase: the 42m diameter European ELT (E-ELT, Gilmozzi 2008), the 30m diameter Thirty Meter Telescope (TMT, Nelson 2008), and the 21-25m diameter Giant Magellan Telescope (GMT, Johns 2008). The step between the current class of telescope and the ELTs is of more than a factor 3 in diameter, larger than what we have experimented in the last $15 \mathrm{yrs}$ when passing from the $4 \mathrm{~m}$ telescopes to the 8-10 meters ones. This contribution is biased in two ways: first it concentrates mostly on the E-ELT instrumentation plan, which is the one the author is more familiar with; second it is biased towards high resolution spectroscopy, because this is the most used technique for deriving detailed chemical abundances. I will show, however, that the three ELTs have quite similar instrumentation plans, and therefore most conclusions and cases valid for the E-ELT can be applied the other ELT telescopes as well.

\section{Why ELTs}

The first advantage of having an ELT is obvious: obtain an enormous photon collecting area. What is perhaps less obvious is that this enormous power can be used not only to reach fainter objects, but also to increase the precision in our spectroscopy.

The second advantage is that if an ELT can work (close to) at the diffraction limit, than we have a tremendous increase in angular resolution; this is capital for several reasons. This implies that we can obtain the highest gain for faint magnitudes because for these objects sky is the main source of noise, and less contribution from sky is recorded. In addition it will be possible to beat confusion, to obtain very high contrast and, for those like me struggling with instrumentation, making the IR spectrographs feasible. In fact, given that $\mathrm{R} \times \alpha \propto \operatorname{tg} \beta \times d_{-}$ins $/ D_{-} t e l$ ), (where $\alpha$ is the aperture in the sky, $\mathrm{R}$ is the resolving power, and $\mathrm{d}_{i n s}$ and $\mathrm{D}_{t e l}$ the instrument and telescope pupils respectively), to obtain for a 1 arcsec seeing Resolving power $\mathrm{R}=100000$ at a $40 \mathrm{~m}$ telescope (and a very steep $\operatorname{tg} \beta=4$ echelle grating) requires an instrument pupil of $4 \mathrm{~m}$ !

It is therefore clear that Adaptive Optics is absolutely essential for ELTs, and indeed a full zoo of AO types is being developed, also in prevision of its use at the ELTs: GLAO, LTAO, MOAO, MCAO, SCAO, XAO are all acronyms to which we will soon be used. 

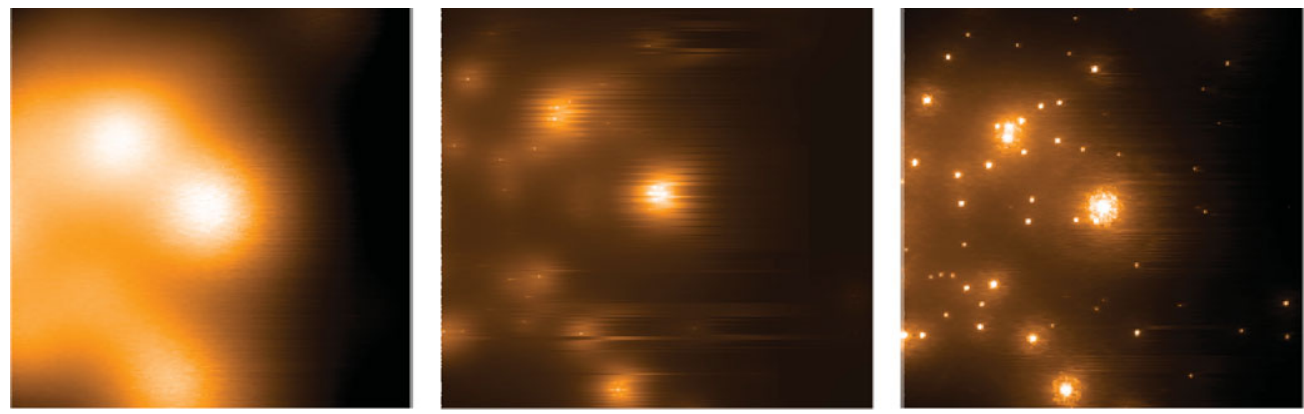

Figure 1. From Evans et al. 2008: EAGLE simulation of the MC cluster NGC346 posed at a distance of $1.3 \mathrm{Mpc}$ as observed by an ELT. The image is $1.6 \times 1.6$ arcsec. The first image the correction is made with Ground Layer AO (GLAO), Multi Object AO (MOAO), and Laser Tomography AO (LTAO).

Figure 1 ( Evans et al. 2008), shows a simulated image of the SMC cluster NGC346 projected at $1.3 \mathrm{Mpc}$, in a $1.6 \times 1.6$ arcsec field, as seen with GLAO, MOAO and LTAO corrections. This figure is very instructive, showing clearly as in the study of stellar population in external galaxies the power of $\mathrm{AO}$ is needed, to beat the confusion at faint magnitudes.

\section{Which Instruments}

The first question in analyzing the instrumentation plans for ELTs, it is to first establish how many will be typically hosted. TMT and GMT have Nasmyth platforms which plan to host several instruments/each; the E-ELT will in addition be equipped with two 'gravity invariant' platforms below the Nasmyth ones, and a coude' focus. Each ELT plans at present to host up to 8-9 instruments simultaneously. "Gedanken Experiments" with realistic dimensions have been made, and showing that it shall be feasible to host 3-4 instruments on the Nasmyth platforms simultaneously.

Figure 2 shows the suite of instruments presently studied for the E-ELT, together with a very brief description and the names of the PIs. As for the VLT most instruments are studied within the ESO community by consortia of institutes.

Figure 3 shows the parameter space covered by the studied instrumentation, in the Field of View vs. wavelength and in the Resolving power vs. wavelength diagrams. The parameter spaces are very well covered; from the diffraction limited instruments (with a typically limited FoV) to the multi-object instruments using the whole 10 arcmin $\mathrm{FoV}$; in resolving power, from the imagers to the two optical and IR high resolutions spectrographs, all the space is covered.

As anticipated above, both TMT and GMT have very similar instrumentation plans, with 9 and 7 instruments respectively. Not all the instrument studied will necessarily be built, and surely not all will be selected for first light. TMT has already chosen their initial complement of 3 instruments (IRIS, IRMS and WFOS), while for the E-ELT and GMT the selection process is expected to be finished within 2010.

\section{High Resolution Spectrographs for the ELT}

It is evident from most contributions to this Symposium that High Resolution (H$\mathrm{R}$ ) spectroscopy is the most widely used and the most powerful technique to determine abundances in the universe. 


\begin{tabular}{l|c}
\hline \multicolumn{1}{c|}{ ACRONYM (P.I.) } & INSTRUMENT TYPE \\
\hline EAGLE (J.G. Cuby) & Wide Field, Multi IFU NIR Spectrograph with MOAO \\
\hline EPICS (M. Kasper) & Planet Imager and Spectrograph with XAO \\
\hline MICADO (R. Genzel) & Diffraction-limited NIR Camera- AO assisted \\
\hline HARMONI (N. Thatte) & Single Field, Wide Band Spectrograph - AO assisted \\
\hline CODEX (L.Pasquini) & High Spectral Resolution, High Stability Visual Spectrograph \\
\hline METIS (B. Brandl) & Mid Infrared Imager \& Spectrograph -AO assisted \\
\hline OPTIMOS (G.Dalton, & Wide Field, Visual, MOS (fibre or slit-based)- GLAO assisted? \\
\hline F.Hammer.-O.LeFevre) & High Spectral Resolution NIR Spectrograph -AO assisted \\
\hline SIMPLE (L. Origlia) & POST -FOCAL ADAPTIVE OPTICS MODULES \\
\hline MAORY (E. Diolaiti) & Multi Conjugate AO module (high Strehl, field up to 2') \\
\hline ATLAS (T. Fusco) & Laser Tomography AO Module (high Strehl, narrow field) \\
\hline
\end{tabular}

Figure 2. List of instruments (with their main characteristics) under phase A study for the E-ELT project. Similar complements are under study for GMT and TMT
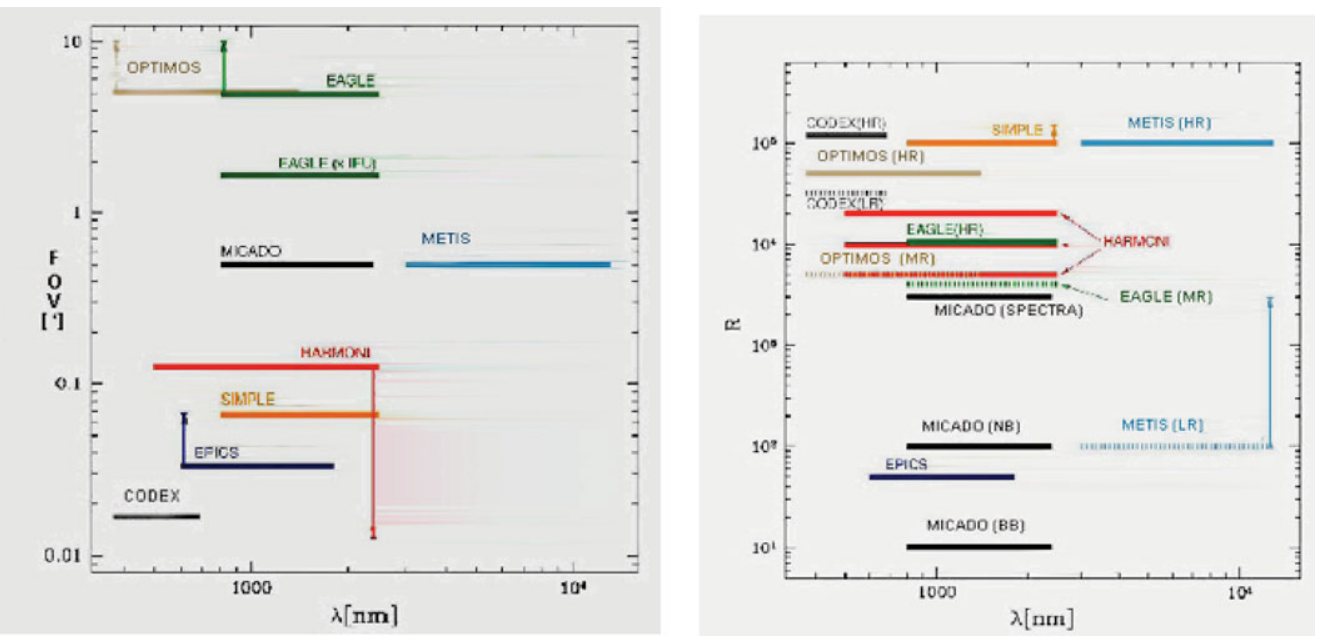

Figure 3. Space covered by the ELT studied instruments: Field of View (left) and Resolving Power (right) vs wavelength. (Courtesy of M. Kissler-Patig)

The E-ELT spectrographs able to provide some substantial high resolution capability are three: CODEX (Pasquini et al. 2005), SIMPLE (Origlia \& Oliva 2009) and OPTIMOS (OPTIMOS web page ).

- CODEX is the optical (range 380-710 nm), high resolution $(\mathrm{R}=120000)$, high 
stability spectrograph. It has an aperture of 0.8 arsceconds in the sky and aims at the highest Doppler precision, $2 \mathrm{cms}^{-1}$ over a period of many years.

- SIMPLE is the AO assisted (aperture on the sky of 0.04 arcseconds) IR high resolution ( $\mathrm{R}$ up to 100000) spectrograph. One of its peculiar characteristics is the very large spectral range (0.9-2.5 $\mu \mathrm{m})$ covered simultaneously in one exposure.

- OPTIMOS/EVE is a Multi-Object fibre optical and IR spectrograph. It has a resolving power from 5000 up to 50000 with a coverage of about $\lambda / 32$ and a central wavelength tunable between 370 and $1700 \mathrm{~nm}$. Thanks to image slicers an aperture of 0.9 arcseconds on the sky is used.

\subsection{IGM and Stars: The faint end}

One of the most interesting aspects of this Symposium has been to link chemical evolution from high redshift to planets. Several speakers have shown how it will be fundamental to push the magnitude limits of objects observed to determine the abundance of intervening IGM. How does the metallicity evolve with redshift? How homogeneous the IGM is ? We need to observed many more high redshift objects to determine the IGM Zn abundance and OPTIMOS will allow to reach QSOs and galaxies as faint as $\mathrm{V}=25$. Another quest is to expand the range of redshifts covered: our present knowledge stops at $\mathrm{Z} \sim 4$ because the Zn lines fall in the IR for higher redshift. With SIMPLE it will be possible to extend these studies at much higher redshifts. With a limiting magnitude around J, H and $\mathrm{K} \sim$ 19, many QSOs will become available for these studies.

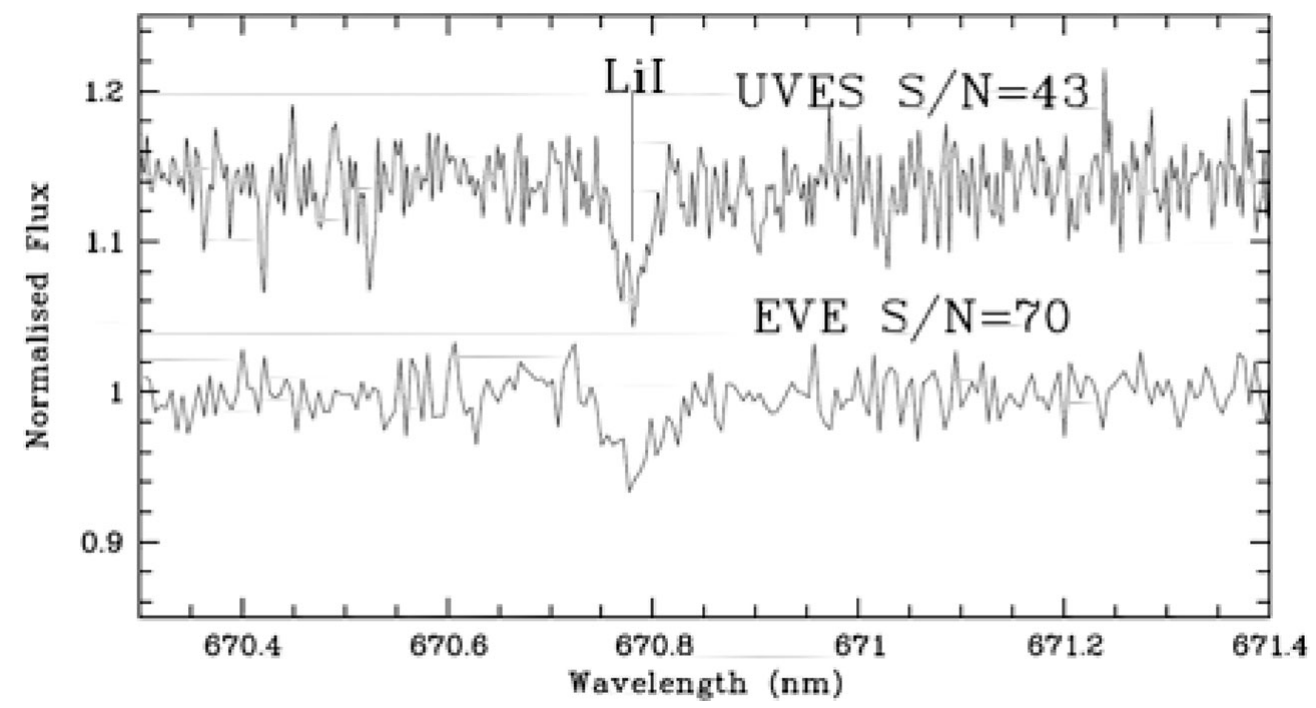

Figure 4. Simulations of Li observations in a bulge main sequence solar star $(\mathrm{V}=21.5)$ with 10 hour of OPTIMOS/EVE at the E-ELT. The spectrum has a quality lower, but comparable with what obtained with VLT+UVES on 17 magnitude stars in the Globular Cluster 47 Tuc (Bonifacio et al. 2007)

For stars as well the E-ELT will represent a tremendous breakthrough, allowing, for instance, to obtain high resolution spectroscopy of low mass stars in the whole Galaxy and in nearby satellites. The understanding of the origin and primordial value of Li, requires, for instance, to study this element in other galaxies and in other populations of our own Galaxy to test whether its abundance in metal poor stars is the same of the Spite plateau (Spite \& Spite 1982) irrespective of the birth place. Figure 4 shows a very exciting simulation of a $\mathrm{Li}$ spectrum of a late type main sequence star $(\mathrm{V}=21.5)$ in the bulge 
as observed by OPTIMOS-EVE at the E-ELT in 10 hours. The spectrum is compared with an observed UVES spectrum of a star in the Globular Cluster 47 Tuc (Bonifacio et al. 2007) and the quality is quite comparable. Observing tens of stars in the bulge it will be possible therefore to derive a detailed history of the evolution of $\mathrm{Li}$, search for correlations with other elements are present in this population. Of course similar studies will be possible for a number of chemical elements. Similarly, it will be possible to obtain $\mathrm{R}=100000$ resolution (and $\mathrm{S} / \mathrm{N}$ ratio 50) spectra for stars up to magnitudes $\mathrm{J}, \mathrm{H}, \mathrm{K}=17$ with SIMPLE.

\subsection{IGM and Stars: The high $S / N$ ratio}

The huge ELT collecting area allows un-precedent high $\mathrm{S} / \mathrm{N}$ ratio and high precision spectroscopy. By acquiring for a sample of objects $\mathrm{S} / \mathrm{N}$ ratio observations as high $\mathrm{s}$ $\mathrm{S} / \mathrm{N}=2000$ with CODEX, it will be possible to study IGM at very low column densities, as low as the median IGM density (NHI 13.5). Figure 4 presents the CODEX simulations for such a case, showing as tiny features will be measurable; an impossible task even for excellent instruments such as UVES at the VLT. By measuring these lines, for instance in QSO pairs or groups it will be possible to distinguish between competing mechanism of IGM enrichment so far proposed.

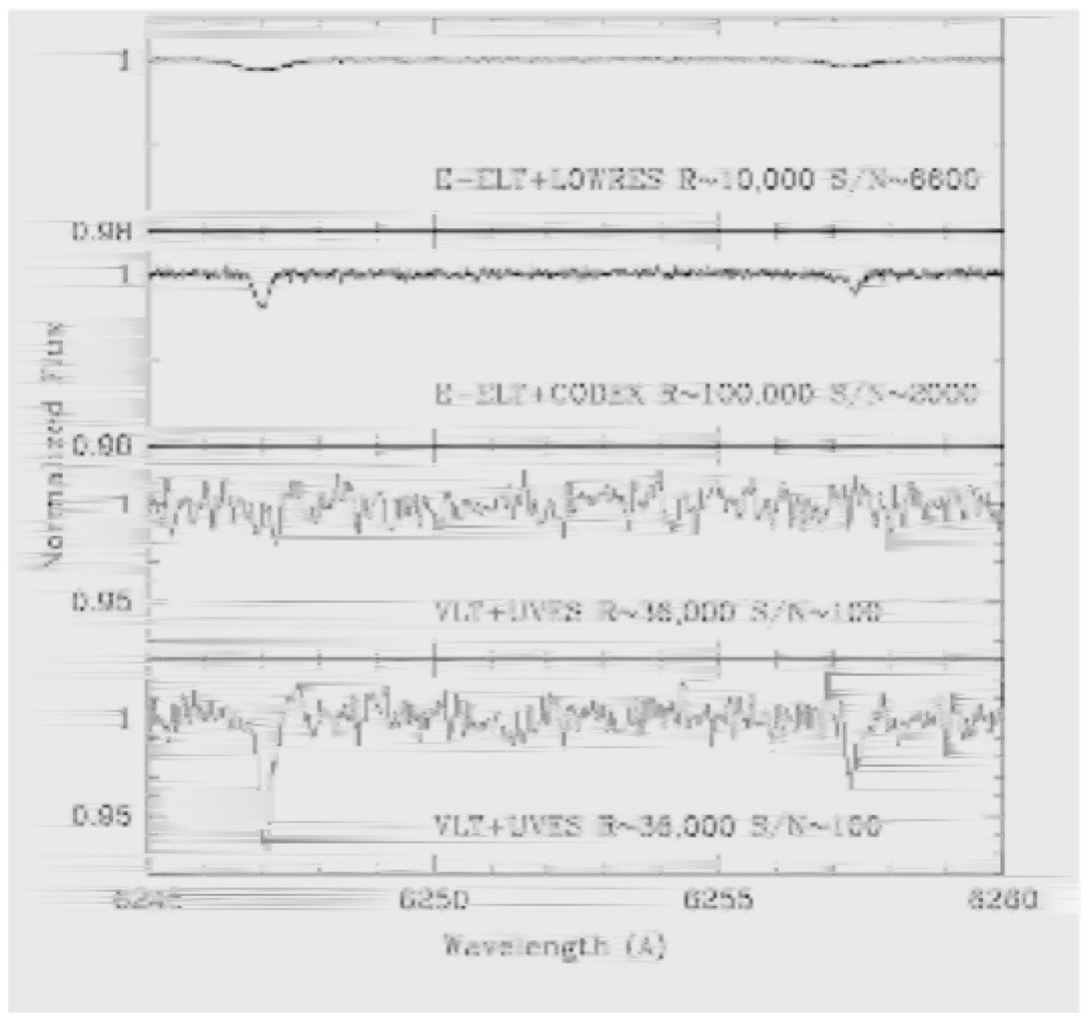

Figure 5. Simulations of IGM CIV lines at different HI column densities. Only with the CODEX resolving power and $\mathrm{S} / \mathrm{N}$ ratio it will be possible to measure metallicity at low IGM density. The last figure below shows the UVES spectrum of CIV corresponding to an NHI density more than 10 times higher than what will be measurable with CODEX. (Courtesy of V. D'Odorico)

For stars one interesting proposal is to use CODEX to determine the age of different galactic populations by determining the age of stars with nucleochronometry. CODEX 
simulations show that with a $\mathrm{S} / \mathrm{N}$ ratio of 1000 it will be possible to determine $\mathrm{Th}$ abundances with an accuracy of 0.03 dex, which translates in an error of 2 Gyrs on the stellar age. In a few hundred hours it shall be possible to obtain ages for hundred of stars in several galactic populations (disks, halo, bulge) and therefore to determine their age with an un-precedent precision, with a method which is completely independent of stellar evolution theory. Similar considerations apply to other interesting isotopic ratios.

\subsection{Exo-planets Atmospheres}

One of the most exciting possibility offered by the ELT is that of studying the chemical composition of exo-planets. What is now possible for only a very few special exo-planets, it will be available for many more. In particular SIMPLE might allow the detection and the analysis of exo-planet atmospheres even for terrestrial objects in habitable zones, provided they are around low mass (M) dwarfs. In this case, it is proposed to study the atmospheric features in absorption, during the transits. The predicted atmospheric absorption features $\left(\mathrm{O}_{2}, \mathrm{CO}_{2}\right)$ have a contrast of $\sim 10^{-5}$ and will be detected when shifted with respect to the earth atmospheric lines up to stars as faint as J, H, K 12.5. Similar cases for brighter planets are proposed by the CODEX and EPICS (Kasper et al. 2008) projects.

\section{Concluding Remarks}

In the ELT era smaller telescopes will still be needed. ELTs will have some limitations and will not be able to perform all kind of observations. FoV is, for example, limited, and in addition the implementation of instruments able to use the full $\mathrm{FoV}$ is usually quite complex. Another example might be UV science: it might not be possible to procure mirror coatings which are extremely efficient both in the IR and at the atmospheric cut off. A second consideration concerns accessibility: there are about a dozen of 6-10 m class telescopes available at present for the community, and they are very often heavily over subscribed. The ELTs will be at most three. We may expect that it will be extremely difficult to obtain large amount of observing time for single programs.

Provided funds are secured, all the three ELT projects are foreseen to start operations around year 2018. We have a very exciting era in front of us!

I would like to thank S. D'Odorico (E-ELT Instrument Project Office), P. Bonifacio (Optimos-EVE), J-G. Cuby (Eagle), P. Hickson (TMT), E. Oliva (Simple), S. Schectman (GMT) for sharing information and figures about their projects.

\section{References}

Bonifacio, P., Pasquini, L., et al. 2007, A\&A , 470, 153

Evans, C. J. \& Lehnert, M. D. 2008, Proc. SPIE, 7014, 201

Gilmozzi, R. 2008, Proc. SPIE, 6986, 698604

Johns, N. 2008, Proc. SPIE, 6986, 698603

Kasper, M. et al. 2008, Proc. SPIE, 7015, 63

Nelson, J. 2008, Proc. SPIE, 6986, 698602

OPTIMOS web page, http://gepi.obspm.fr/les-projets/instrumentation-e-elt/article/optimoseve?lang=en

Origlia, L. \& Oliva, E. 2005, Earth, Moon and Planets, 105, 123

Pasquini, L. et al. 2005, The Messenger, 122, 10

Spite, F. \& Spite, M. 1982, A\&A, 115, 357 